\title{
Automated registration for augmenting micro-CT 3D images
}

S. J. Latham ${ }^{1} \quad$ T. K. Varslot ${ }^{2} \quad$ A. P. Sheppard ${ }^{3}$

(Received 31 July 2008; revised 20 November 2008)

\begin{abstract}
Micro-CT imaging allows probing of material 3D structure down to the micrometre scale. However, often there exists structure at the sub-micrometre scale which significantly influences the macrophysical properties of the material. One possible solution for mitigating this micro-CT resolution limitation is to incorporate information from higher resolution Back-scattered Scanning Electron Microscopy (BSEM) imaging techniques. A first step toward incorporating this high resolution data into micro-CT models is to align the BSEM 2D image(s) with the corresponding region(s) of the micro-CT 3D image. This article presents an automated multi-start multi-resolution parallel registration algorithm which has been successfully used to achieve accurate alignment of BSEM and micro-CT image pairs.
\end{abstract}

http://anziamj.austms.org.au/ojs/index.php/ANZIAMJ/article/view/1389 gives this article, (c) Austral. Mathematical Soc. 2008. Published December 16, 2008. ISSN 1446-8735. (Print two pages per sheet of paper.) 


\section{Contents}

1 Introduction

C535

2 Registration algorithm

C536

2.1 Algorithm steps . . . . . . . . . . . . . . . . C537

2.2 Parallel implementation . . . . . . . . . . . . . . C539

3 Image alignment results

C539

4 Powell local optimization

C544

5 Discussion

C545

References

C547

\section{Introduction}

The study of X-ray micro-CT tomograms provides a variety of information regarding pore and matrix structure of a rock core. However, a complete analysis is often impeded because there exist significant regions in the core sample which contain pore or rock-matrix features below the micro-CT imaging resolution. Almost every carbonate rock and any rock or soil containing clay contains micropores smaller than 100 nanometres, which are key to understanding seismic and transport properties. Micro-CT analysis is enhanced by combining complementary information from other imaging techniques with the micro-CT data in an attempt to describe the core structure at micro, meso and macro scales [4]. BSEM imaging is capable of resolving features at the nanometre scale and hence offers the opportunity to resolve micropore structure. Here we consider the first step in utilizing this high resolution BSEM data, which is to accurately align the BSEM $2 \mathrm{D}$ image with the corresponding region of the micro-CT 3D image. The accurate alignment allows direct vi- 
sual comparison of image features and provides a means of visually assessing the quality of the micro-CT image. In addition to this qualitative advantage, there is the potential for quantitative benefit by associating micro-CT grey levels with a porosity calculated from the BSEM images.

This article presents a registration algorithm which is a generalised and parallelised form of that used by Jenkinson and Smith [2] to register 3D images of the brain. The algorithm solves a global optimization problem via a multi-start multi-resolution approach and the $\mathrm{C}++$ implementation uses the Message Passing Interface to take advantage of high performance computer architectures.

\section{Registration algorithm}

Solutions to the image registration problem are well represented in the literature and a recent survey of techniques can be found in the reviews by Brown [1] and Zitová [6]. To register a BSEM image with the corresponding micro-CT image, this article adopts the common approach of formulating the alignment task as a global optimization problem. Fundamental to this formulation is a cost function or distance metric which gives a quantitative value indicating the merit of the alignment between two images with respect to a number of transform parameters. BSEM images are very similar to $\mathrm{X}$-ray projection images, since both methods are primarily sensitive to the density of the electron cloud surrounding each atom. Because of this similarity in image modality, the correlation coefficient metric has proven to be a sufficient discriminator of alignment for BSEM and micro-CT image pairs. BSEM 2D images represent a thin planar slice through the 3D core sample and therefore a rigid transformation should be sufficient to align the BSEM image within the micro-CT image. Here, the similarity transformation was used as the allowable deformation. The similarity transform consists of a rigid transformation plus an isotropic scaling. The scaling degree of freedom 
(DOF) accounts for small errors in pixel/voxel size estimates. The optimization problem is thus defined to minimize the negative correlation coefficient with respect to seven parameters/DOF (three translational, three rotational and an isotropic scaling).

A global minimization algorithm is required to solve the optimization problem. In general, registration cost functions contain many local minima which precludes the exclusive use of local descent methods. In this article, a multi-resolution approach is combined with an exhaustive low resolution search. At the lowest resolution the exhaustive search produces a small set of transformation parameters, at least one of which is within the capture radius of a global minimum. These initial sets of transformation parameters are used as starting solutions for a local numerical optimization method (a variation of Powell's direction set algorithm [5]) to further refine and reduce the transformation parameter sets at increasing resolutions until the final best transformation parameters are determined at the highest resolution.

\subsection{Algorithm steps}

1. Pre-process images. The reconstructed tomographic 3D images are preprocessed to identify any voxels which are not contained with the core sample region. These voxels are assigned a mask-attenuation value. Voxels with mask-attenuation value do not contribute to the evaluation of the distance metric. Lower resolution BSEM images generally contain distortion which is removed during pre-processing. All BSEM images are then combined via $2 \mathrm{D}$ registration into a mosaic. Pixels in the BSEM image which do not lie within the core sample region are also assigned a mask-attenuation value.

2. Exhaustive search at lowest resolution. Set search index $s=0$. The pair of images are down sampled by a power-of-two factor $d_{0}$, which is determined by the size of the features present in the images. The results in this article used a maximum down sample factor of $d_{0}=16$ in each 
dimension so that a $2048^{3}$ voxel image is down sampled to generate a $128^{3}$ voxel image. An exhaustive search is conducted by evaluating the distance metric at points which form a regular grid in the transformation parameter space. The translational step length is that of a down sampled voxel side, and the rotational step size is two degrees. In this step, the rotational DOF are restricted to the $z$-axis as BSEM images are generated from thin sections taken (approximately) from the $x y$-plane, and the micro-CT images are generated from samples which have their cylindrical axis aligned with the $z$-axis. The scaling parameter is held constant at one, as the tomogram voxel side lengths and BSEM pixel side lengths are generally known to within $\pm 3 \%$ and this scaling error is not significant for the highest image down sampling. Keep the $\mathrm{N}_{0}$ sets of transformation parameters, which gave the $\mathrm{N}_{0}$ lowest values of the distance metric. These $\mathrm{N}_{0}$ parameter sets are used as starting solutions for the iterative local optimization.

3. $s=s+1, d_{s}=d_{s-1}$.

4. Check if finished. If $d_{s}<1$ then go to Step 7 .

5. Perform local mathematical optimizations. Create new image pair by down sampling the original image pair by a factor of $d_{s}$. For each of the $\mathrm{N}_{s-1}$ best transformation parameters, perform a local optimization with the solution from the previous step as the starting point. Keep the $N_{s}$ sets of transformation parameters, which gave the $N_{s}$ lowest values of the distance metric.

6. Update down sample factor. $s=s+1, d_{s}=d_{s-1} / 2$, go to Step 4 .

7. Finished. The best transformation parameters from the previous step are the final solution giving the optimal alignment of the original fixed and moving images. 


\subsection{Parallel implementation}

In order for the above global minimization method to be computationally feasible, there are two parallelisation strategies which are used when implementing the steps in software on high performance Non-Uniform Memory Access architectures. The first is a task parallelism strategy where individual tasks are performed independently on computational units. The second strategy is a data parallelism approach where each computational unit contains only a subset of the discrete image pair data. Task parallelism is of great advantage when calculating the distance metric values in Step 2. At low resolutions each computational unit evaluates the distance metric for a subset of the transformation parameter grid points independently of other computational units. When all computational units have evaluated their subset of values, there is a result collection stage where the subsets of values are gathered from each of the computational units in order to rank the best transform parameters. Similarly, task parallelism is used to advantage in Step 5 for the lower resolution images, where each computational unit performs a subset of the iterative local optimizations, independently of other computational units. For the higher resolution images, the data parallel strategy is preferred. In this strategy, each computational unit only contains a subset of the discrete image data. Step 5 is then performed using a Master/Worker division of labour. The master computational unit controls the iterative optimization algorithm while each worker computational unit calculates a portion of the total distance metric result based on the subset of image data held in its local RAM.

\section{Image alignment results}

To obtain high resolution BSEM images from within the volume imaged by micro-CT, we used the following experimental protocol. The sample, usually a cylinder with a diameter of $5 \mathrm{~mm}$, was initially imaged in the micro-CT. 
The same sample was then cut perpendicular to the axis of the cylinder and one half was impregnated with epoxy. The impregnated half was then polished down to form a thin section with a thickness of approximately $30 \mu \mathrm{m}$. The thin section was finally mounted on a glass slide. This is a destructive process which precludes re-imaging of the sample. BSEM images of core sample thin sections were obtained at a number of resolutions, (for the samples shown here, magnifications factors were $40 \times, 450 \times$ or $1000 \times)$. For the lowest magnification, a series of BSEM scans was performed so that the entire area of the core sample thin section is imaged. These lowest resolution BSEM 2D images were individually pre-processed to remove spatial distortion. Spatial distortion is inherent in the BSEM image acquisition apparatus. The spatial distortion removal process used a BSEM image of a known grid to calculate a warp field which is subsequently used to correct the BSEM images. The separate BSEM thin section images were then registered to form a single montage image of the entire thin section. This $2 \mathrm{D}$ registration was performed using a phase correlation frequency domain technique (Kuglin and Hines [3] or Brown [1] give descriptions), which is highly efficient at determining translational shifts between images. The result of this $2 \mathrm{D}$ registration is a mosaic image typically containing approximately $4000 \times 4000$ pixels. The montage image was then registered with the micro-CT $3 \mathrm{D}$ image by employing our multi-start multi-resolution algorithm, with the correlation coefficient used as the distance metric and the similarity transformation defining the seven DOF of the optimization problem. The higher resolution BSEM images were registered with the BSEM montage image and then overlaid with the corresponding region of the registered micro-CT image.

Figure 1 shows BSEM images (left column) of two carbonate samples with the registered region of their corresponding micro-CT image (right column). The left column contains montage images formed by performing 2D registration on the de-warped 'quadrants' BSEM images. The right image of the first row is the corresponding slice of the micro-CT 3D image $(2.59 \mu \mathrm{m}$ voxel size). The alignment between the two images is good, with a correlation coefficient of 0.601 . Figure 1 highlights the similarity of the BSEM images and the 

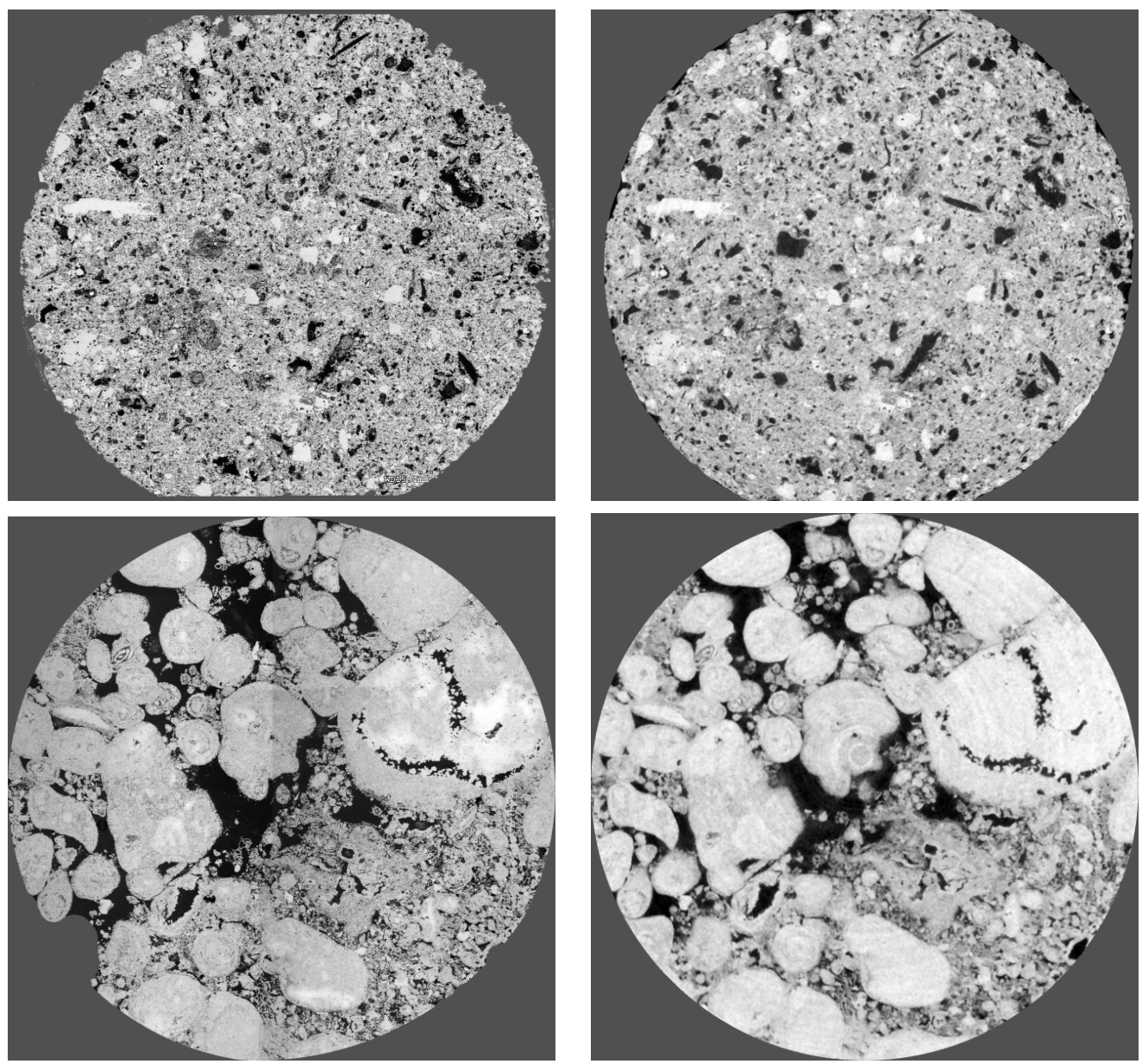

FIGURE 1: BSEM images of carbonate samples (left column) and registered region of corresponding micro-CT 3D image (right column). 

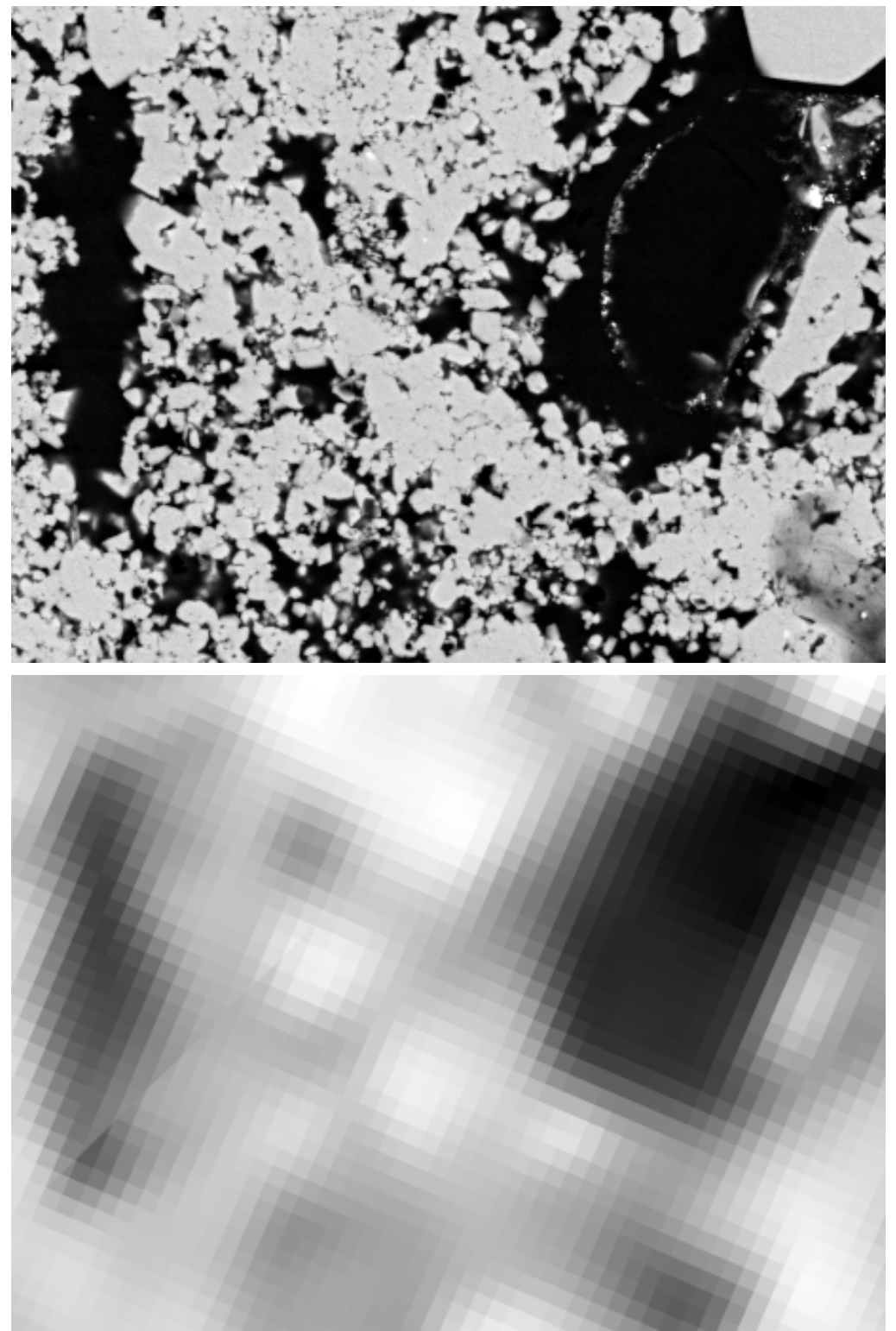

FiguRE 2: High resolution $1000 \times$ magnification BSEM image (top) and registered region of micro-CT image (bottom) from the sample shown in the top row of Figure 1. 

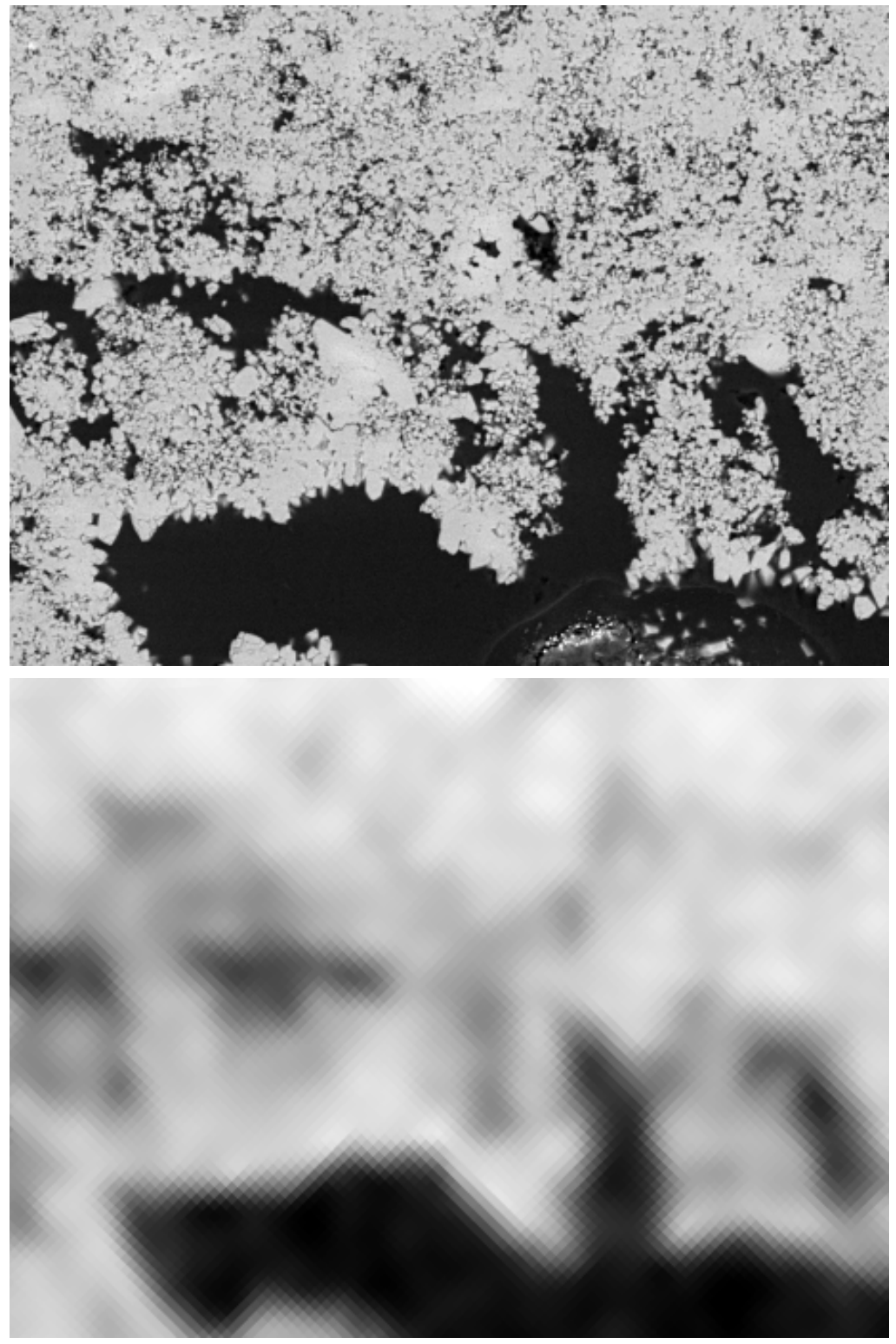

FIGURE 3: High resolution $450 \times$ magnification BSEM image (top) and registered region of micro-CT image (bottom) from the sample shown in the bottom row of Figure 1. 
reconstructed X-ray projection images, a result of the fact that both techniques calculate image intensities which are primarily related to the density of the electron cloud surrounding each atom. There are noticeable differences between the two images in the top row of Figure 1 where some of the larger empty pore spaces in the micro-CT image appear to have non-empty counterparts in the BSEM image. Presumably, there has been some disruption to the sample during the thin section preparation. The bottom row of Figure 1 shows good alignment between the montage BSEM image on the left (40 $\times$ magnification, $1.24 \mu \mathrm{m}$ pixel size) and the micro-CT image slice on the right $(2.69 \mu \mathrm{m}$ voxel size $)$ with a correlation coefficient of 0.716 . Figure 2 shows the $1000 \times$ magnification BSEM image (top), from the sample in the top row of Figure 1 and the corresponding region of the micro-CT 3D image (bottom). The high resolution BSEM image clearly illustrates features (porosity) which are not easily classifiable in the micro-CT image. The upper image in Figure 3 is a $450 \times$ magnification BSEM image $(0.110 \mu \mathrm{m}$ pixel size) of the sample from the bottom row of Figure 1 and the lower image is the corresponding region of the micro-CT image.

\section{Powell local optimization}

A modified Powell direction-set local optimization method was used in the registration procedure [5]. The implementation uses Brent's method [5] for the line minimization. The Powell method does not require cost function gradients and is relatively simple to implement. While it is possible to approximate correlation coefficient gradients, with respect to the seven similarity transformation DOF, we have opted for a non-gradient optimization method in order to simplify the implementation and to allow for the immediate evaluation of distance metrics (and/or transformation parameterisations) where the gradients are not so easily estimated.

The stopping/convergence criteria for the Powell iteration was specified 


\begin{tabular}{|l|ccccc|}
\hline & $d_{1}=16$ & $d_{2}=8$ & $d_{3}=4$ & $d_{4}=2$ & $d_{5}=1$ \\
\hline Registration 1 & $7.0[4,16]$ & $4.0[3,4]$ & $4.5[4,6]$ & $8.1[6,13]$ & $5.0[5,5]$ \\
Registration 2 & $5.6[4,15]$ & $4.6[4,7]$ & $6.7[5,12]$ & $10.3[6,14]$ & $5.0[5,5]$ \\
\hline
\end{tabular}

TABLE 1: Powell iteration count statistics: mean, minimum and maximum iterations shown for each resolution with down sample factor $d_{s}, s=1, \ldots, 5$.

as a relative tolerance for the change in distance metric value between Powell (outer) iterations. For the full resolution optimization (search $s=5$ ), this tolerance was set to $\tau_{5}=\tau=10^{-8}$, which is (approximately) equivalent to four significant figures of accuracy in each of the transformation DOF. For the lower resolution optimizations, the tolerance for search $s$ is made less stringent by setting the stopping criterion to be $\tau_{s}=\tau d_{s}{ }^{2}$. In addition to the relative tolerance stopping criterion, the iterative optimization was stopped when the number of iterations exceeded 16. Table 1 presents iteration statistics for the Powell method used to register the images pairs of Figure 1 (Registration 1 data is for the top row image pair and the Registration 2 data is for the bottom row image pair). For search $s=1,2,3,4,5$, the number of local optimizations $\mathrm{N}_{s-1}$ were $\mathrm{N}_{0}=64, \mathrm{~N}_{1}=32, \mathrm{~N}_{2}=16$, $\mathrm{N}_{3}=8$ and $\mathrm{N}_{4}=1$.

\section{Discussion}

Our registration method uses an exhaustive search at a down-sampled resolution in order to determine similarity transformation parameters which are within the global minimum capture radius of the correlation coefficient distance metric. The exhaustive search is computationally feasible in this case because of four factors:

1. feature size in the images allow a significant down-sample factor; 
2. the use of task-parallelism to concurrently compute correlation coefficient values;

3. using a priori knowledge about the orientation of the thin section used to generate the BSEM image in order to reduce the size of the search space; and

4. only a small percentage $(\approx 15 \%)$ of BSEM image pixels need be used to approximate the correlation coefficient and this greatly reduces the time taken to evaluate the distance metric.

The registration runs in less than an hour on 64 CPUs and consumes less than $32 \mathrm{~Gb}$ of RAM when aligning a $4000^{2}$ BSEM image with a $2048^{3}$ voxel micro-CT image.

While the results of Section 3 describe alignment of rock sample images, the algorithm has the potential to be utilised in other areas. Different types of specimen (biological, medical, etc.) can potentially benefit from the comparison of the aligned BSEM and micro-CT image pair. Furthermore, with modification to the distance metric, the technique applies to the registration of the dry core sample micro-CT 3D image with the micro-CT 3D image of the same core sample flooded with a contrast agent. Several flooding experiments can be conducted (under varying wettability conditions) and the subsequent series of registered micro-CT 3D images be used to compare and analyse the fluid partitioning.

Acknowledgements We thank the Australian Research Council and the member companies of the Digital Core Laboratory for providing their funding support and also acknowledge the Australian Partnership for Advanced Computation for supplying the HPC resources used to produce the registrations presented in this article. 


\section{References}

[1] L. G. Brown. A survey of image registration techniques. ACM Computing Surveys, 24:325-376, 1992. http://portal.acm.org/citation.cfm?id=146370.146374 C536, C540

[2] Mark Jenkinson and Stephen Smith. A global optimisation method for robust affine registration of brain images. Medical Image Analysis, 5:143-156, June 2001. doi:10.1016/S1361-8415(01)00036-6 C536

[3] C. D. Kuglin and D. C. Hines. The phase correlation image alignment method. In Proc. Int. Conf. on Cybernetics and Society, volume 4, pages $163-165,1975$. C540

[4] G.S. Padhy, C. Lemaire, E.S. Amirtharaj, and M.A. Ioannidis. Pore size distribution in multiscale porous media as revealed by DDIF-NMR, mercury porosimetry and statistical image analysis. Colloids and Surfaces A: Physicochemical and Engineering Aspects, 300:222-234, June 2007. doi:10.1016/j.colsurfa.2006.12.039 C535

[5] William H. Press, Brian P. Flannery, Saul A. Teukolsky, and William T. Vetterling. Numerical Recipes in C: The Art of Scientific Computing. Cambridge University Press, 2nd edition, October 1992. http://www.nrbook.com/a/bookcpdf.php C537, C544

[6] B. Zitová and J. Flusser. Image registration methods: a survey. Image and Vision Computing, 21:977-1000, 2003. doi:10.1016/S0262-8856(03)00137-9 C536

\section{Author addresses}

1. S. J. Latham, Department of Applied Mathematics, Research School of Physics and Engineering, Australian National University, 
ACT, Australia.

http://www.rsphysse.anu.edu.au/appmaths

mailto:shane.latham@anu.edu.au

2. T. K. Varslot, Department of Applied Mathematics, Research School of Physics and Engineering, Australian National University, ACT, Australia.

http://www.rsphysse.anu.edu.au/appmaths

3. A. P. Sheppard, Department of Applied Mathematics, Research School of Physics and Engineering, Australian National University, ACT, Australia. 